\title{
Modelling Chlamydia Trachomatis Infection Among Young Women in Ghana: A Case Study at Tarkwa Nsuaem Municipality
}

\author{
Christiana Cynthia Nyarko*, Nicholas Nicodemus Nana Nsowa-Nuamah, Peter Kwesi Nyarko, \\ Eric Neebo Wiah, Albert Buabeng
}

Department of Mathematical Sciences, Faculty of Engineering, University of Mines and Technology, Tarkwa, Ghana

Email address:

ccnyarko@umat.edu.gh (C.C. Nyarko)

${ }^{*}$ Corresponding author

To cite this article:

Christiana Cynthia Nyarko, Nicholas Nicodemus Nana Nsowa-Nuamah, Peter Kwesi Nyarko, Eric Neebo Wiah, Albert Buabeng. Modelling Chlamydia Trachomatis Infection Among Young Women in Ghana: A Case Study at Tarkwa Nsuaem Municipality. American Journal of Applied Mathematics. Vol. 9, No. 3, 2021, pp. 75-85. doi: 10.11648/j.ajam.20210903.13

Received: May 8, 2021; Accepted: June 3, 2021; Published: June 16, 2021

\begin{abstract}
Chlamydia Genital infection has been a global health issue especially among most developing countries. Although, a lot of researchers have modelled CT infection to determine the impact of different intervals between Chlamydia infection and the development of Pelvic Inflammatory Disease (PID) on the cost-effectiveness of screening and the use of Chlamydia vaccine. This paper seeks to model the dynamics of Chlamydia Trachomatis (CT) infection among females who were diagnosed of vaginal discharge and the likelihood of developing PID complications. The model was formulated using a sexual network to explore the relationship between Chlamydia infection through diagnosed vaginal infection and PID. A sample of 147 females were diagnosed and screened of Chlamydia related symptoms on a routine check-up in the Tarkwa Nsuaem Municipality in the Western part of Ghana. Lyapunov functions was used to prove the necessary and sufficient conditions for Stability State of the system while Next Generation Method was also used to calculate the basic reproduction number $\left(R_{0}\right)$. The Stability Analysis of the Modified SIRS model shows that the system is locally and asymptotically stable at the Disease-Free Equilibrium (DFE) $\mathrm{E}_{0}$, when $R_{0}<1$, and when $R_{0}>1$, the Endemic Equilibrium (EE) $E^{*}$, was found to be locally and asymptotically stable at certain conditions. It was observed that, as the distribution increases sharply at a given contact rate $(\beta)$ of 0.05 , many of the patients were infected within the first three days as compared to when the contact rate was 0.001 . Moreover, at contact rates $(\beta)$ of $0.5, R_{0}$ was greater than one, this shows how CT infection spreads in the population using parameter values in Table 1 . Thus, the effects of change in the various initial conditions of the parameters $(\lambda)$ and $(\beta)$ on vaginal discharge and PID infections, turn to increase sharply at a higher infection rate for the first ten days of infection especially with vaginal discharge and then become stable over a period of time. This confirms the incubation period which is usually 7 to 10 days of infection. The paper concludes that, young women aged 18-24 years are more at risk of Chlamydia Trachomatis infection if diagnosed of vaginal discharge or PID and suggest early medication which is highly subsidised will help curb the spread of CT infection in the Municipality.
\end{abstract}

Keywords: Chlamydia, Discharge, Infection, PID, Trachomatis, Equilibrium, Endemic, Simulation

\section{Introduction}

Chlamydia Trachomatis (CT) infection is arguably one of the most common Sexually-Transmitted Infections (STIs) worldwide $[1,2]$. It is prevalent in both industrialised and developing countries worldwide. The World Health Organisation (WHO) estimated 100.4 million cases of CT infection world-wide in 2008 with 92 million cases in Africa. In 2005, Africa reported a prevalence rate of $3.9 \%$ among females and $2.4 \%$ in males. CT infection is observed to be higher in rural areas of some African countries [3]. According to Stamm [4], Chlamydia is more prevalent in heterosexuals than in homosexuals and has a higher incidence in those less than 20 years particularly among Africans. In Ghana, prevalence rate of $\mathrm{CT}$ infection ranges from $3 \%$ to $10.1 \%$ 
among female sex workers in Accra [5, 6]. The incidence of the disease is not well-defined because in most countries CT infections are not noticeable, and mostly are asymptomatic in nature, and thus tend to escape detection $[2,7]$. Untreated Chlamydia infection could lead to severe reproductive complications especially in females and about $10-20 \%$ of female infertility is associated with tubal factor infertility. Although CT infection will effectively clear in most women, the infection may persist in some and could ascend to the upper genital tract leading to Pelvic Inflammatory Disease (PID) infection [8].

\subsection{Chlamydia Trachomatis Infections in Women}

The asymptomatic nature of Chlamydia Trachomatis (CT) infection in most women makes it difficult to attract adequate medication and in most cases could easily lead to severe reproductive complications especially in young adult females. Hence, about $10-20 \%$ of female infertility is associated with tubal factor infertility [8 ]. In 201150 million women were globally infected with Chlamydia trachomatis and 34 million in Sub-Saharan Africa and Southeast Asia (WHO; Adachi et al., [9, 10])

Genital CT infection in women usually appears in the form of vaginal discharge which develops into chronic subclinical infection. However, those at risk of the infection are found among young sexually active females who are virtually asymptomatic and so do not seek treatment [2]. According to Molano et al. [11] women infected with serogroup B and C types had a longer duration of genital chlamydial infection which untreated could usually leads to Pelvic Inflammatory Disease (PID). However, PID is known to be a syndrome associated with the transmission of micro-organisms from the vagina and cervix to the endometrium, salpingeal tubes and adjacent structures Hussen et al., [3]. Smith et al., [12] studied the progression from Chlamydia infection to PID complications and the cost-effectiveness of Chlamydia screening. However, in this paper, we modelled the progression of chlamydia infection from vaginal discharge to PID complications and minimising its spread through early medication.

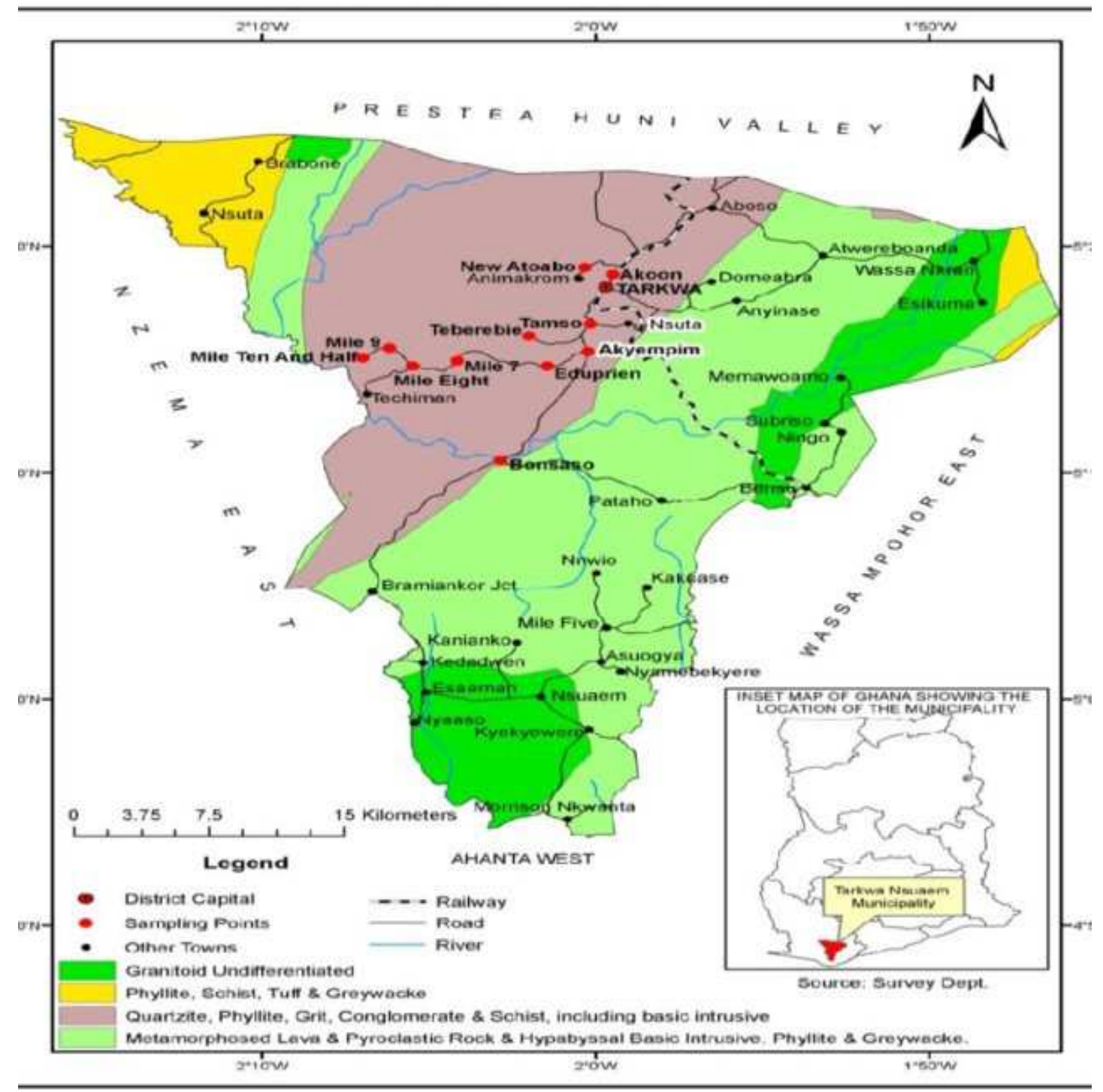

Figure 1. A Map Showing the Study Area. 


\subsection{Study Area}

The study area is the Tarkwa Nsuaem Municipality (TNM) which covers a land area of about $2,354 \mathrm{sq} . \mathrm{km}$. It is situated in the central part of the region and shares common boundaries with Prestea-Huni Valley District to the north, with Ahanta West District to the south, with Nzema East district to the west and with Mpohor Wassa East District to the east. The Municipality lies in the evergreen rainforest belt and records a mean annual rainfall of $110 \mathrm{~mm}$. The Municipality has a total land area of 905.2 and it is one of the richest in terms mineral resource in Ghana. According to the Ghana population and housing census 2010, the total population of the Tarkwa Nsuaem Municipality is 90477 which represents $48.43 \%$ female and $51.57 \%$ male. One of the major economic activities in the area is surface mining and illegal small-scale mining popularly called "Galamsey". There are multi-national companies engaged in the mining of gold and manganese. These companies, which are mostly manned by elite whites, employ Ghanaians from all over the country as well as natives of other African countries. Hence, the municipality consists of migrants of different cultures and backgrounds. The presence of these migrants attracts all kinds of sex workers from other neighboring countries. The activities of these sex workers increase the rate of CT infection and other STIs in the Municipality. The map of the study area is show in Figure 1.

\subsection{Sources of Data}

Study participants were selected from three hospitals in the Tarkwa Nsuaem Municipality (TNM). The hospitals include; Tarkwa Government hospital, Goldfield's hospital and Ghana Manganese Company (GMC) Hospital Tarkwa. A laboratory screening test for 200 patients in the Municipality were employed to determine the presence of Chlamydia Trachomatis infection in symptomatic men and women in the municipality through screening [2].

\section{Mathematical Modelling of Chlamydia Infection}

\subsection{Model Formulation}

In this paper a deterministic mathematical model was formulated to describe the modelling of Chlamydia Trachomatis (CT) infection among female patients in TNM. This population is further compartmentalized into epidemiological classes as shown as Figure 2.

The paper considered only female patients for constructing the compartmental model, the susceptible $S(t)$ group consist of female patients screened for Chlamydia infection without treatment. Number of female patients infected $I(t)$, reflect Chlamydia prevalence in the study population and $R(t)$ represents female patients who had recovered from CT infections due to treatment at rate $\rho$. The study differentiated between Chlamydia infection due to vaginal discharge and PID by categorising the infectious period into two stages; $\left(I_{V D}\right)$ represents infected patients with vaginal discharge and $\left(I_{P I D}\right)$, indicates transmission from vaginal discharge to PID infection. Hence, infectious state, $I=I_{V D}+I_{P I D}$.

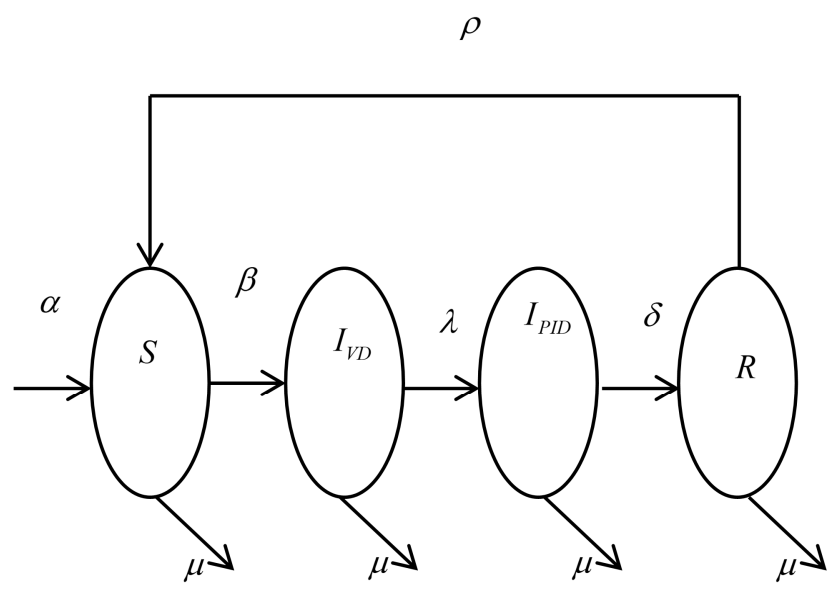

Figure 2. Compartmental Model of Vaginal Discharge Dynamics.

The detailed transitions between these four classes are depicted in figure 2. The susceptible class $(\mathrm{S})$ is increased either by birth or immigration at a rate alpha $(\alpha)$. It is decreased through contact with an infected individual at rate beta $(\beta)$, and by natural death at rate mu $(\mu)$. The infectious class $(I)$ is generated through infection of the susceptible. The infectious class is of two folds, Chlamydia infection due to vaginal discharge symptoms which decreases by natural death at rate $(\mu)$ and further progresses to the second stage of PID complications as a result of untreated vaginal discharge at rate lambda $(\lambda)$. Patients in this class recover with temporal immunity at rate delta $(\delta)$. The recruitment rate of the population is $\mu$ and the total number of new births is denoted by $\mu \mathrm{N}$. The contact rate $\beta$, is the rate at which susceptible individuals come into contact with the infected population. The recovery rate $\gamma$, is the rate of progression from the infectious class to the recovery class.

\subsection{Model Assumptions}

This model assumes a homogeneous mixing of the population with individual patient having equal likelihood of being infected with Chlamydia, if they come into contact with an infected male. The study considered the following assumptions before the model equations were formulated. The population under consideration is fixed. In this model, a constant recruitment rate $\alpha$ to the susceptible population per unit time is considered. There is a natural mortality rate $(\mu)$ in all the classes. Since the above models are dealing with humans, all the state variables are assumed to be positive, that is $t \geq 0$ It is also assumed that, the recruitment rate $\alpha$ is equal to a natural death rate $(\mu)$ at the Disease-Free Equilibrium (DFE) point. 


\subsection{Equations of the Chlamydia T Model}

Using the above assumptions, the following systems of differential equations are formulated from the compartmental diagram above to mimic the dynamics of CT infection of a female patient as presented in Equation (1):

$$
\left\{\begin{array}{l}
\frac{d S(t)}{d t}=\alpha-\beta S(t) I_{V D}(t)-\mu S(t)+\rho R(t) \\
\frac{d I_{V D}(t)}{d t}=\beta S(t) I_{V D}(t)-(\lambda+\mu) I_{V D}(t) \\
\frac{d I_{P I D}(t)}{d t}=\lambda I_{V D}(t)-(\delta+\mu) I_{P I D}(t) \\
\frac{d R(t)}{d t}=\delta I_{P I D}(t)-(\rho+\mu) R(t)
\end{array}\right.
$$

The total population at time (t) is given by $N(t)=S(t)+I_{V D}(t)+I_{P I D}(t)+R(t)$. The systems of ordinary differential equations formulated above are nonlinear with initial conditions $S(0)=s_{0}, I_{V D}(0)=i_{1,0}, I_{P I D}(0)=i_{2,0}, R(0)=\rho_{0}$.

\subsection{Methods and Materials}

This paper used next generating matrix method to determine the expression for the basic reproductive number $\left(R_{0}\right)$. The equilibrium stability of Ordinary Differential Equations (ODE's) was determined by the sign of real part eigenvalues of the Jacobian matrix. Birkhof and Rota's theorem among others and functions were used to develop the mathematical modelling of CT infections.

\subsection{Invariant Region}

It is assumed that all the state variables and parameters of the model are non-negative for all $t \geq 0$ since the model is dealing with population. The system of equations (1) was analysed in a suitable feasible region where all state variables are positive.

Theorem 1. The solutions of the system of equations (1) are contained in the region $\phi=\Phi$

Proof. Let $\left[S(t), I_{V D}(t), I_{P I D}(t), R(t)\right] \in \mathbb{R}_{+}^{4}$ be any solution of the system of Equations (1), with non-negative initial conditions. Then, it can be deduced that:

$$
\begin{aligned}
& \frac{d N(t)}{d t} \leq \alpha-\mu N, \\
& \frac{d N(t)}{d t}+\mu N \leq \alpha,
\end{aligned}
$$

Using the Integrating Factor, (IF), IF $=e^{\mu \int d t}=e^{\mu t}$, Multiplying (2) by the integrating factor we obtain;

$$
\begin{aligned}
& e^{\mu t} \frac{d N(t)}{d t}+\mu N(t) e^{\mu t} \leq \alpha e^{\mu t}, \\
& \frac{d}{d t}\left(N e^{\mu t}\right) \leq \alpha e^{\mu t},
\end{aligned}
$$

now integrating on both sides gives;

$$
N(t) e^{\mu t} \leq \frac{\alpha}{\mu} e^{\mu t}+C
$$

where $\mathrm{C}$ is the constant of integration. Hence,

$$
N(t) \leq \frac{\alpha}{\mu}+C e^{-\mu t},
$$

Applying initial conditions, when $t=0, N(t)=N(0)$. Implies,

$$
N(0)-\frac{\alpha}{\mu} \leq C
$$

Comparing (3) and (4) gives:

$$
N(t) \leq \frac{\alpha}{\mu}+\left(N(0)-\frac{\alpha}{\mu}\right) e^{-\mu t},
$$

Then, applying Birkhof and Rota's theorem on the differential inequality as $t \rightarrow \infty$, it can be obtained that $0 \leq N(t) \leq \frac{\alpha}{\mu}$. Hence all feasible solutions of equation (1) enter the region

$$
\Phi=\left\{\left[S(t), I_{V D}(t), I_{P I D}(t), R(t)\right]: N(t) \leq \frac{\alpha}{\mu}\right\}
$$

Hence the system of Equation (1) is both mathematically and epidemiologically meaningful.

\subsection{Positivity of Solutions}

The positivity and boundedness of solutions of Equation (1) is established, which imply that the model is well posed. Therefore, the discussion below focuses on the conditions under which the model being studied has non-negative solutions.

Theorem 2. Given the initial conditions $S(0), I_{V D}(0), I_{P I D}(0)$ and $R(0)$, the system of differential equations (1) is non-negative, and its solutions remain bounded and are non-negative for all $t \geq 0$.

Proof. To prove the theorem, the model system of differential equations (1) will be used. The first equation of the model (1), can be deduced as:

$$
\frac{d S(t)}{d t}=\alpha-\beta S(t) I_{V D}(t)-\mu S(t)+\rho R(t)
$$

To determine the positivity of $S(t)$, consider $\frac{d S(t)}{d t} \leq \alpha-\mu S(t)$, consequently,

$$
\frac{d S(t)}{d t}+\mu S(t) \leq \alpha
$$


This is a first order homogeneous differential equation. It's Integrating Factor (IF), thus $I F=e^{\int \mu d t}=e^{\mu t}$. Multiplying by the $I F$ on both sides yields

$$
e^{\mu t} \frac{d S(t)}{d t}+\mu S(t) e^{\mu t} \leq \alpha e^{\mu t} .
$$

It follows that $d\left[e^{\mu t} S(t)\right] \leq \alpha e^{\mu t} d t$. Integrating on both sides yields

$$
e^{\mu t} S(t) \leq \frac{\alpha}{\mu} e^{\mu t}+c
$$

where $\mathrm{c}$ is the constant of integration, it then follows that

$$
S(t) \leq \frac{\alpha}{\mu}+e^{-\mu t} c
$$

Applying initial conditions, when $t=0, S(t)=S(0)$, then

$$
S(0)-\frac{\alpha}{\mu} \leq c .
$$

Hence $S(t) \leq \frac{\alpha}{\mu}+c e^{-\mu t}$, then $S(t)>0$ if $t=0$ and $t \rightarrow \infty$.

From equation two of the system of differential equations (1), it can be deduced that, to determine the positivity of $I_{V D}(t)$, consider

$$
\begin{array}{r}
\frac{d I_{V D}(t)}{d t} \geq-(\mu+\gamma) I_{V D}(t), \text { consequently, } \\
\frac{d I_{V D}(t)}{d t}+(\mu+\gamma) I_{V D}(t) \geq 0 .
\end{array}
$$

This is a first order homogeneous differential equation. Its Integrating Factor (IF)

$$
I F=e^{\int(\mu+\gamma) d t}=e^{(\mu+\gamma) t}
$$

Multiplying by the $I F$ on both sides yields

$$
e^{(\mu+\gamma) t} \frac{d I_{V D}(t)}{d t}+(\mu+\gamma) I_{V D}(t) e^{(\mu+\gamma) t} \geq 0 .
$$

It follows that $d\left(e^{(\mu+\gamma) t} I_{V D}(t)\right) \geq 0 d t$. Integrating on both sides yields $e^{(\mu+\gamma) t} I_{V D}(t) \geq c$, where $c$ is the constant of integration, it then follows that $I_{V D}(t) \geq e^{-(\mu+\gamma) t} c$. Applying initial conditions, when $t=0, I_{V D}(t)=I_{V D}(0)$, then $I_{V D}(0) \geq c$.

Hence $I_{V D}(t) \geq e^{-(\mu+\gamma) t} \mathrm{c}$, and $I_{V D}(0) \geq 0$, then $I_{V D}(t) \geq 0$ if $t=0$ and $t \rightarrow \infty$.

Similarly, for the remaining variables the same procedure can be used to show that they are positive for all $t \geq 0$.

\subsubsection{Equilibrium Solutions (Steady States)}

The steady states of the system of differential Equation (1) is obtained by setting or equating each of the four nonlinear equations to zero as shown in Equation (6)

$$
\left\{\begin{array}{l}
\alpha-\beta S(t) I_{V D}(t)-\mu S(t)+\rho R(t)=0 \\
\beta S(t) I_{V D}(t)-(\lambda+\mu) I_{V D}(t)=0 \\
\lambda I_{V D}(t)-(\delta+\mu) I_{P I D}(t)=0 \\
\delta I_{P I D}(t)-(\rho+\mu) R(t)=0
\end{array}\right.
$$

Solving (6), there exist two equilibrium solutions: Disease free equilibrium and an Endemic equilibrium.

\subsubsection{Disease Free Equilibrium (DFE) Solution}

At the Disease-Free Equilibrium (DFE) all the system for the nonlinear differential equations is set to zero except the susceptible compartment which is assumed to be present as a result of birth, $\alpha$ death $\lambda$ which is always part of the population. So, at the DFE, substituting $I_{V D}(t)=I_{P I D}(t)=R(t)=0$ in the system of Equation (1), the system reduces to: $\alpha-\mu S(t)=0$ which gives $S(t)=\frac{\alpha}{\mu}$

Therefore, the disease-free equilibrium (DFE) denoted by $E_{0}$ of the system of differential equation (1) gives

$$
E_{0}=(S(t), 0,0,0)=\left(\frac{\alpha}{\mu}, 0,0,0\right)
$$

The disease-free equilibrium will only exist when $\mu>0$. Again, when $\alpha=\mu=1$ the DFE becomes (7):

$$
E_{0}=(1, \quad 0, \quad 0, \quad 0)^{T}
$$

\subsubsection{Endemic Equilibrium Solution}

The endemic equilibrium (EE) $E^{*}$ of the model is also obtained by setting the system of equations again to zero. The DEE is given by the following equations

$$
E^{*}=\left\{S^{*}(t), I_{V D}^{*}(t), I_{P I D}^{*}(t), R^{*}(t)\right\}
$$

where

$$
\begin{aligned}
& S^{*}(t)=\frac{\mu+\lambda}{\beta} \\
& I_{V D}^{*}(t)=(\delta+\mu) I_{P I D}^{*} \\
& I_{P I D}^{*}(t)=\frac{\lambda\left(\alpha \beta-\mu \lambda-\mu^{2}\right)(\rho+\mu)}{\beta \mu\left(\mu \delta+\lambda \delta+\rho \delta+\mu^{2}+\mu \lambda+\mu \rho+\rho \gamma\right)} \\
& R^{*}(t)=\frac{\lambda \delta\left(\alpha \beta-\mu \lambda-\mu^{2}\right)}{\beta \mu\left(\mu \delta+\lambda \delta+\rho \delta+\mu^{2}+\mu \lambda+\mu \rho+\rho \lambda\right)}
\end{aligned}
$$

The endemic equilibrium solution is always positive, as all the parameters are considered to be positive. The natural mortality rate $\mu$, is considered constant throughout the model. 


\subsection{Determination of the Basic Reproduction Number $\left(R_{0}\right)$}

In order to assess the stability $\left(E_{0}\right)$ of the system, the basic reproductive number $\left(R_{0}\right)$ was derived, using the next generation method by Diekmann and Heesterbeek [9] on the system of differential equations (1). The $R_{0}$ at the DFE was calculated by taking the dominant eigenvalue (spectral radius) of the matrix partial derivatives as presented in (8)

$$
\left(\frac{\partial F_{i}}{\partial x_{j}}\left(E_{0}\right)\right)\left(\frac{\partial V_{i}}{\partial x_{j}}\left(E_{0}\right)\right)^{-1}
$$

where $F_{i}$ is the rate of appearance of new infection in compartment $I_{i}, V_{i}^{+}$is the transfer of women into compartment $I_{i}, V_{i}^{-}$is the transfer of women out of the compartment $I_{i}$ by all other means, $x_{j}$ is $\left[\left(S(t), I_{V D}(t), I_{P I D}(t), R(t)\right]^{T} \quad\right.$ and $\quad E_{0} \quad$ is the interior equilibrium.

$$
\left(\begin{array}{l}
f_{1} \\
f_{2}
\end{array}\right)=\left(\begin{array}{c}
\beta S(t) I_{V D}(t) \\
0
\end{array}\right)
$$

where $i=1,2$

The associated matrix at the disease-free equilibrium is given by computing the infected compartments as presented in Equation (9)

$$
\begin{aligned}
F=\frac{\partial \underline{F}}{\partial x_{j}} & =\left[\begin{array}{ll}
\frac{\partial f_{1}}{\partial I_{V D}}\left(E_{0}\right) & \frac{\partial f_{1}}{\partial I_{P I D}}\left(E_{0}\right) \\
\frac{\partial f_{2}}{\partial I_{V D}}\left(E_{0}\right) & \frac{\partial f_{2}}{\partial I_{P I D}}\left(E_{0}\right)
\end{array}\right] \\
F & =\left(\begin{array}{cc}
\frac{\alpha \beta}{\mu} & 0 \\
0 & 0
\end{array}\right),
\end{aligned}
$$

and

$$
\left(\begin{array}{c}
v_{1} \\
v_{2}
\end{array}\right)=\left(\begin{array}{c}
(\lambda+\mu) I_{V D} \\
-\lambda I_{V D}(t)+(\delta+\mu) I_{P I D}(t)
\end{array}\right)
$$

The associated matrix at the disease-free equilibrium is given by Equation (10)

$$
V=\frac{\partial \underline{V}}{\partial X_{j}}=\left[\begin{array}{ll}
\frac{\partial v_{1}}{\partial I_{V D}}\left(E_{0}\right) & \frac{\partial v_{1}}{\partial I_{P I D}}\left(E_{0}\right) \\
\frac{\partial v_{2}}{\partial I_{V D}}\left(E_{0}\right) & \frac{\partial v_{2}}{\partial I_{P I D}}\left(E_{0}\right)
\end{array}\right]
$$

which gives,

$$
V=\frac{\partial V}{\partial x_{j}}=\left(\begin{array}{cc}
\lambda+\mu & 0 \\
-\lambda & \delta+\mu
\end{array}\right)
$$

with

$$
V^{-1}=\left(\begin{array}{cc}
\frac{1}{\lambda+\mu} & 0 \\
\frac{\lambda}{(\lambda+\mu)(\delta+\mu)} & \frac{1}{\delta+\mu}
\end{array}\right)
$$

therefore,

$$
\begin{gathered}
F V^{-1}=\left(\begin{array}{cc}
\frac{\alpha \beta}{\mu} & 0 \\
0 & 0
\end{array}\right)\left(\begin{array}{cc}
\frac{1}{\lambda+\mu} & 0 \\
\frac{\lambda}{(\gamma+\mu)(\delta+\mu)} & \frac{1}{\delta+\mu}
\end{array}\right) \\
F V^{-1}=\left(\begin{array}{cc}
\frac{\alpha \beta}{\mu(\lambda+\mu)} & 0 \\
0 & 0
\end{array}\right)
\end{gathered}
$$

The spectral radius of matrix $F V^{-1}$ is $\rho\left(F V^{-1}\right)=\frac{\beta \alpha}{\mu(\lambda+\mu)}$. Hence this is in line with theory that, the basic reproduction number $\mathrm{R}_{0}$ is the dominant eigenvalue of the matrix partial derivatives $F V^{-1}$ as presented in Equation (8) which is $R_{0}=\frac{\beta \alpha}{\mu(\lambda+\mu)}$.

\subsection{Stability of Disease-Free Equilibria}

This section presents the stability of the disease-free equilibrium. The Lyapunov function is used to prove the local and global stabilities of equilibria.

\subsubsection{Local Asymptotic Stability of the Disease-Free Equilibrium}

Theorem 3. The Disease-Free Equilibrium (DFE) $E_{0}$ of the system of differential Equation (1) is locally asymptotically stable if $R_{0}<1$.

Proof. The Jacobian matrix $\mathrm{J}$ of the system of differential equations (1) is given by

$$
J=\left(\begin{array}{cccc}
-\beta I_{V D}-\mu & -\beta S & 0 & \rho \\
\beta I_{V D} & \beta S-\mu-\lambda & 0 & 0 \\
0 & \lambda & -\delta-\mu & 0 \\
0 & 0 & \delta & -\rho-\mu
\end{array}\right)
$$

Evaluating matrix $\mathrm{J}$ at the disease-free equilibrium, $E_{0}$ 
gives Equation (13).

$$
J_{E 0}=\left(\begin{array}{cccc}
-\mu & -\frac{\beta \alpha}{\mu} & 0 & \rho \\
0 & \frac{\beta \alpha}{\mu}-\mu-\lambda & 0 & 0 \\
0 & \lambda & -\delta-\mu & 0 \\
0 & 0 & \delta & -\rho-\mu
\end{array}\right)
$$

The characteristic equation resulting from the Jacobian matrix is

$$
\sigma^{4}+a_{3} \sigma^{3}+a_{2} \sigma^{2}+a_{1} \sigma+a_{0}=0
$$

where $a_{0}=\left(\mu \lambda-\beta \alpha+\mu^{2}\right)\left(\delta \mu+\delta \rho+\rho \mu+\mu^{2}\right)$

$$
\begin{gathered}
a_{1}=\left(\gamma+2 \mu-\frac{\beta \alpha}{\mu}\right)\left(\delta \mu+\delta \rho+\rho \mu+\mu^{2}\right)+\left(\mu \lambda+\mu^{2}-\beta \alpha\right)(\delta+2 \mu+\rho) \\
a_{2}=\left(\lambda+2 \mu-\frac{\beta \alpha}{\mu}\right)(\delta+2 \mu+\rho)+\left(\mu \lambda+\mu^{2}-\beta \alpha\right)+\left(\delta \mu+\delta \rho+\rho \mu+\mu^{2}\right) \\
a_{3}=\left(\delta+\lambda+4 \mu+\rho-\frac{\beta \alpha}{\mu}\right)
\end{gathered}
$$

The matrix $J_{E_{0}}$ has eigenvalues

$$
\begin{gathered}
\sigma_{1}=-\mu<0, \sigma_{2}=-\delta-\mu<0, \sigma_{2}=-\rho-\mu<0, \\
\sigma_{4}=-\frac{-\beta \alpha+\mu(\lambda+\mu)}{\mu}
\end{gathered}
$$

Clearly $\sigma_{1}, \sigma_{2}$ and $\sigma_{3}$ are negative and for DFE to be locally asymptotically stable, if $R_{0}<1$ all real eigenvalues must be negative [10]. Thus, it is required that $\sigma_{4}<0$. Therefore, for local asymptotic stability at DFE we have;

$$
-\frac{-\beta \alpha+\mu(\lambda+\mu)}{\mu},
$$

This is possible provided

$$
\beta \alpha<\mu(\lambda+\mu) \Rightarrow \frac{\beta \alpha}{\mu(\lambda+\mu)}<1 \text {. But } \frac{\beta \alpha}{\mu(\lambda+\mu)}=R_{0}
$$

Hence, the disease-free equilibrium $E_{0}$ is locally asymptotically stable, since $R_{0}<1$.

\subsubsection{Global Asymptotic Stability of the Disease-Free Equilibrium}

Theorem 4. The Disease-Free Equilibrium (DFE) $E_{0}$ of the system of differential Equation (1) is globally asymptotically stable whenever $R_{0}<1$.

Proof. Constructing the Lyapunov function of the form

$$
\begin{gathered}
\dot{L}=u_{1} S+u_{2} I_{V D}+u_{3} I_{P I D}+u_{4} R \\
=u_{1}\left(\alpha-\beta S I_{V D}-\mu S+\rho R\right)+u_{2}\left[\beta S I_{V D}-(\lambda+\mu) I_{V D}\right] \\
+u_{3}\left[\lambda I_{V D}-(\delta+\mu) I_{P I D}\right]+u_{4}\left[\delta I_{P I D}-(\rho+\mu) R\right]
\end{gathered}
$$

where $u_{1}, u_{2}, u_{3}$ and $u_{4}$ are positive constants to be estimated. By principle of differentiation with respect to $t$, we have

$$
\begin{gathered}
\dot{L}=u_{1}\left(\alpha-\beta S I_{V D}-\mu S+\rho R\right)+u_{2}\left[\beta S I_{V D}-(\lambda+\mu) I_{V D}\right] \\
+u_{3}\left[\left(\lambda I_{V D}-(\delta+\mu) I_{P I D}\right]+u_{4}\left[\delta I_{P I D}-(\rho+\mu) R\right]\right. \\
\dot{L}=u_{1} \alpha-u_{1} \beta S I_{V D}-u_{1} \mu S+u_{1} \rho R+u_{2} \beta S I_{V D}-u_{2} \lambda I_{V D}-u_{2} \mu I_{V D} \\
+u_{3} \lambda I_{V D}-u_{3} \delta I_{P I D}-u_{3} \mu I_{P I D}+u_{4} \delta I_{P I D}-u_{4} \rho R-u_{4} \mu R \\
\dot{L}=\beta S I_{V D}\left(u_{2}-u_{1}\right)+\lambda I_{V D}\left(u_{3}-u_{2}\right)+\delta I_{P I D}\left(u_{4}-u_{3}\right) \\
\\
\rho R\left(u_{1}-u_{4}\right)+\left[u_{1} \alpha-\mu\left(u_{4} R+u_{1} S+u_{2} I_{V D}+u_{3} I_{P I D}\right)\right]
\end{gathered}
$$

Now if we choose the constants $u_{1}=u_{2}=u_{3}=u_{4}=1$ we then obtain $-(\mu N-\alpha)<0$. Thus, the disease-free equilibrium of the Lyapunov function is globally asymptotically stable for $R_{0}<0$.

\subsubsection{Stability of Disease Endemic Equilibria}

This paper presents the stability of the endemic equilibrium using the local stability as examined by Lyapunov functional methods.

\subsubsection{Local Asymptotic Stability of the Endemic}

\section{Equilibrium}

Theorem 5. The Endemic Equilibrium $E^{*}$ of the system of Equation (1) is locally asymptotically stable if $R_{0}>1$ under some conditions.

Proof. The variation matrix of the system of differential Equation (1) at $E^{*}=\left(S^{*}(t), I_{V D}^{*}(t), I_{P I D}^{*}(t), R^{*}(t)\right)$ is given by Equation (17),

$$
V_{E^{*}}=\left[\begin{array}{cccc}
-\beta I_{V D}^{*}-\mu & -\mu S^{*} & 0 & \rho \\
\beta I_{V D}^{*} & \beta S^{*}-\mu-\lambda & 0 & 0 \\
0 & \lambda & -\delta-\mu & 0 \\
0 & 0 & \delta & -\rho-\mu
\end{array}\right]
$$

Therefore, the characteristic equation of $V_{E^{*}}$ is given by (18):

$$
\sigma^{4}+A_{1} \sigma^{3}+A_{2} \sigma^{2}+A_{3} \sigma+A_{4}=0
$$

where,

$$
A_{1}=\beta\left(-S^{*}+I_{V D}^{*}\right)+3 \mu+\lambda+\delta+\rho
$$

$$
\left.A_{2}=\lambda\left(\delta S^{*}-3 \mu S^{*}-\rho S^{*}+\rho I_{V D}^{*}+\lambda I_{V D}^{*}+3 \mu I_{V D}^{*}\right) 4 \mu+3 \delta+2 \lambda-\rho-1\right)+\gamma \delta+\lambda \rho+\delta \rho
$$




$$
\begin{aligned}
A_{3}= & \lambda \mu\left(2 \delta S^{*}-3 \mu S^{*}-2 \rho S^{*}+2 \rho I_{V D}^{*}+2 \lambda I_{V D}^{*}+3 \mu I_{V D}^{*}+\delta I_{V D}^{*}\right)+\rho \delta \beta\left(S^{*}+I_{V D}^{*}\right)+\lambda \beta\left(\delta I_{V D}^{*}+\rho I_{V D}^{*}\right) \\
& \mu^{2}(3 \delta-\rho+3 \mu+1)+\lambda \mu(2 \delta+2 \mu+2 \rho)+\delta \rho(2 \mu+\lambda) \\
A_{4}= & \rho \lambda \mu\left(\delta S^{*}-\mu S^{*}+\delta I^{*}+I^{*}+I^{*}\right)+\beta \mu\left(\mu \delta S^{*}-\mu^{2} S^{*}+\mu^{2} I^{*}+\mu \lambda I^{*}+\mu \delta I^{*}+\lambda \delta I^{*}\right) \\
& +\mu^{3}(\delta+\lambda+\rho+\mu)+\rho \mu(\mu \delta+\lambda \delta+\mu \lambda)+\mu^{2} \lambda \delta
\end{aligned}
$$

By the Routh-Hurwitz criterion [13], it follows that all eigenvalues of the characteristic Equation (19) has negative real parts if and only if

$$
\begin{aligned}
A_{i}>0, i=1,2,3,4, \quad D_{1}=A_{1}>0, \\
D_{2}=\left|\begin{array}{cc}
A_{1} & A_{3} \\
1 & A_{2}
\end{array}\right|=A_{1} A_{2}-A_{3}>0 \\
D_{3}=\left|\begin{array}{ccc}
A_{1} & A_{3} & 0 \\
1 & A_{2} & A_{4} \\
0 & A_{1} & A_{3}
\end{array}\right|=A_{1} A_{2} A_{3}-A_{1} A_{4}-A_{3}^{2}>0 \\
D_{4}=\left|\begin{array}{cccc}
A_{1} & A_{3} & 0 & 0 \\
1 & A_{2} & A_{4} & 0 \\
0 & A_{1} & A_{3} & 0 \\
0 & 0 & A_{2} & A_{4}
\end{array}\right|=A_{1} A_{2} A_{3} A_{4}-A_{1}^{2} A_{3} A_{4}-A_{3}^{2} A_{4}>0
\end{aligned}
$$

Therefore, the system of differential equations (1) again shows that the endemic equilibrium $\left(E^{*}\right)$ is locally asymptotically stable, when $R_{0}>1$. Hence the conditions in (19) are satisfied.

\section{Analysis and Simulation of the SIRS (S IVG IPID RS) Model}

A numerical simulation of the system of Equation (1) was carried out using a set of parameter values in Table 1 to illustrate some of the analytical results of the study. The parameter values used were obtained from literature and other parameters that were not available in literature were estimated. The study used 2010 Population and Housing Census for Ghana to estimate the average life expectancy for age groups from 10 years to $95+$ years. The total population was $21,727,772$ million with an average life expectancy of 64.22 years [14]. Therefore, the average overall mortality rate is estimated as $\mu=1 / 64.22=0.016$ with a recruitment rate of $\alpha=0.04$.

\subsection{Numerical Simulation of the Model}

The stability on the two equilibrium points: The diseasefree equilibrium state and the endemic state depend mainly on the basic reproductive ratio $R_{0}$. Therefore when $R_{0}=0.260<1$, the system is stable and when $R_{0}=1.235>1$, the system is unstable as contact rate increases to 0.8 . The basic reproduction ratio $\left(R_{0}\right)$ was simulated and calculated based on the estimated parameters from literature, see Table 1.

Table 1. Description of Parameters Values for the Model.

\begin{tabular}{llll}
\hline Parameter & Description & Parameter range & Source \\
\hline$\alpha$ & Recruitment rate & $0.05-1.12$ & {$[15]$} \\
$\beta$ & Contact rate & $0.001-0.5$ & Estimated \\
$\lambda$ & Rate at which infected individuals progress to PID complications & $0.001-0.7$ & 0.016 \\
$\mu$ & Natural mortality rate & Estimated & Estimated \\
\hline
\end{tabular}

\subsection{Numerical Simulations of the Modified SIRS (S IVG IPID RS) Model}

The following graphs are the solution curves of the system of differential Equation (1) using a set of reasonable parameter values estimated in Table 1.

\subsection{Sensitivity Analysis of Model Parameters}

Sensitivity analysis was performed to determine the effect of small perturbations on parameter values in the initial value of the system. Changes in sensitive parameter values have significant effect on the spread of a disease from one person to another. A numerical sensitivity index was computed to help single out parameters that have a high impact on and which should be targeted by intervention strategies and its impact on the reproductive number $\left(R_{0}\right)$. The sensitivity index is defined as the normalised forward sensitivity index of a variable, w, that differentiably depends on a parameter $\mathrm{H}$;

$$
\Upsilon_{H}^{w}=\frac{\partial w}{\partial H} \times \frac{H}{w}
$$


The sensitivity of $R_{0}$ to each of the four different parameters described in Table 1. Thus, the sensitivity index of $R_{0}$ with respect to $\beta$, is derived as $\Upsilon_{\beta}^{R_{0}}=\frac{\partial R_{0}}{\partial \beta} \times \frac{\beta}{R_{0}}=1.000$ other indices include $\Upsilon_{\alpha}^{R_{0}}, \Upsilon_{\mu}^{R_{0}}$ and $\Upsilon_{\lambda}^{R_{0}}$.

The various model parameters were examined against $\mathrm{CT}$ infection with vaginal discharge and PID, to check which of the parameters is more sensitive, see figures 4 to 7 .

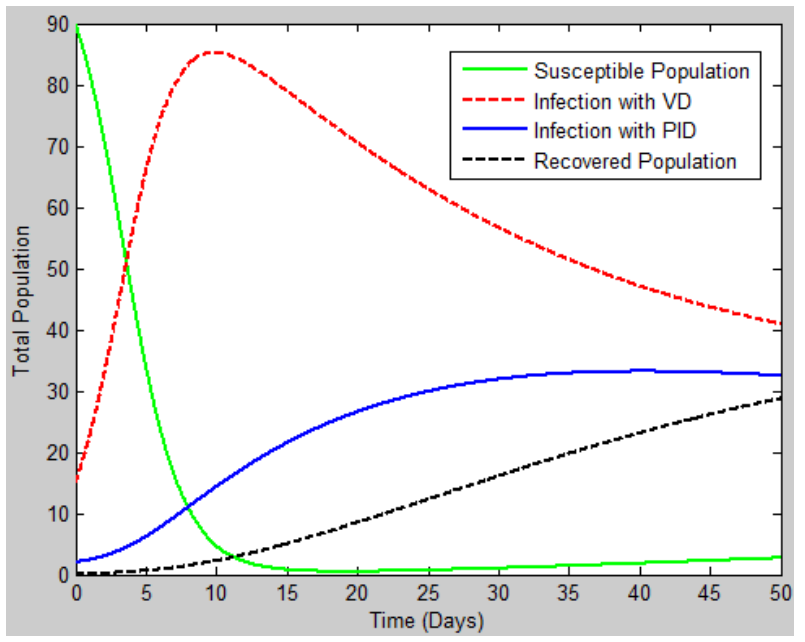

Figure 3. Interaction curves of the Model.

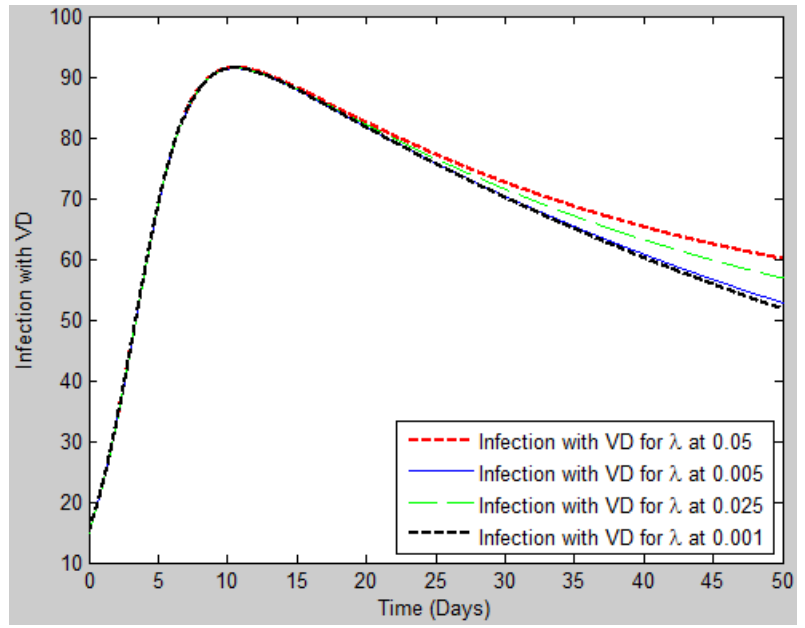

Figure 4. Infected Patients with Vaginal Discharge (VD) at various Initial Conditions of Lambda $(\lambda)$.

The system of equations (1) was simulated to depict the dynamics of the entire model given an initial population of ninety thousand four hundred and seventy - seven (90477) against fifty days' time period. It was found that in figure 3 , the susceptible population decreases considerably at the onset of the infection and moves asymptotically as time increases. The infectious class with vaginal discharge starts to increase exponentially and then falls gradually until it reaches its equilibrium position whilst, the infectious class with PID starts to increase slowly and then increases over time, and then maintains some level of equilibrium. Similarly, the recovery class increases slightly and maintains some level of stability as time increases. This depicts the likelihood of many of the patients diagnosed of vaginal discharge with CT infection progressing to PID infections and infecting other new partners through sexual contact or from mother to child.

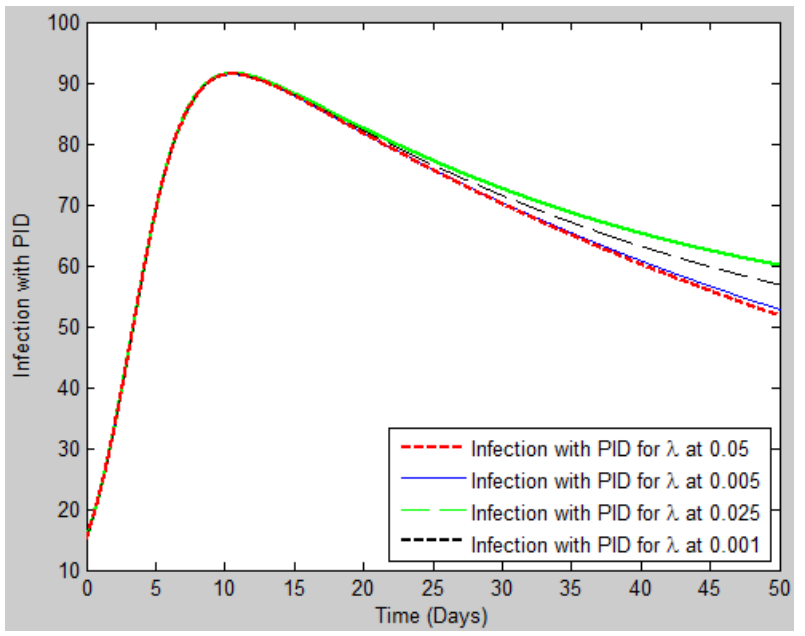

Figure 5. Infected Patients with PID at various Initial Conditions of Lambda $(\lambda)$.

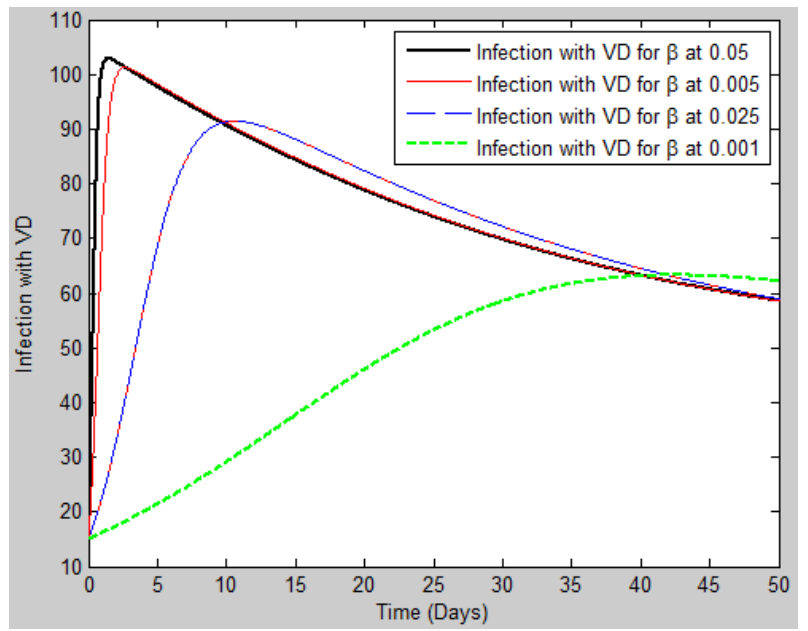

Figure 6. Infected Patients with Vaginal Discharge (VD) at various Initial Conditions of Beta $(\beta)$.

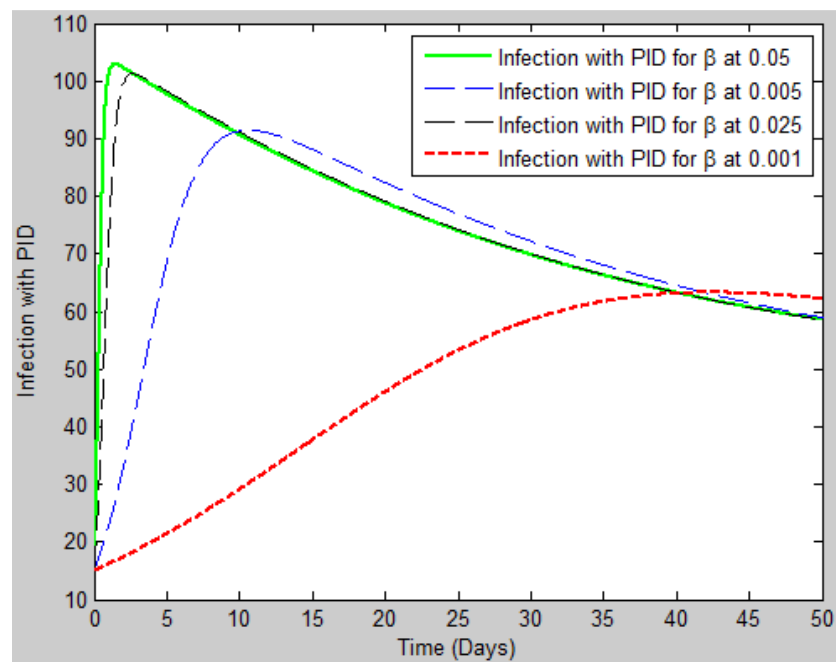

Figure 7. Infected Patients with PID at Various initial Conditions of Beta $(\beta)$. 


\subsection{Discussions of the Model}

Sensitivity of the parameters were analysed and arranged from most sensitive to least. The most sensitive parameters are the recruitment rate and the contact rate ( $\alpha$ and $\beta$ respectively) and the least sensitive parameter is the death rate $(\mu)$. The parameter $\beta$ and $\lambda$ was used as control and simulate the dynamics of $R_{0}$.

Figures 4 to 5 show the effect of the changes in the various initial conditions of the parameters, $(\lambda)$ and $(\beta)$, on Chlamydia infection with vaginal discharge and PID. It was observed that, as the distribution increases sharply at a given contact rate of 0.05 , many of the patients were infected within the first three days compare to when the contact rate is 0.001 . In figures 4 and 5 , the effects of change in the various initial conditions of the parameters $(\lambda)$, on Chlamydia infection on the symptoms, turn to increase sharply at a higher infection rate for the first ten days of infection especially with vaginal discharge and then become stable over a period of time. This confirms the incubation period which is usually 7 to 10 days of infection.

However, any attempt to reduce mostly the contact rate is marked by a reduction in the number of infected individuals. At $\beta=0.001$ to $0.05, R_{0}<1$, it can be observed that, the infection minimizes drastically. Moreover, at contact rates of $\beta=0.5, R_{0}>1$, this shows how CT infection spreads in the population given parameter values in Table 1 .

\section{Conclusion}

In this paper, a deterministic mathematical model for CT infection with respect to vaginal discharge and PID have been formulated and its dynamics duly investigated. The basic reproduction number was derived from the system of equations formulated. The disease-free equilibrium, $E_{0}$, was calculated and found to be locally asymptotically stable whenever $R_{0}<1$. The endemic equilibrium $\mathrm{E}^{*}$ was also found to be locally asymptotically stable whenever $R_{0}>1$. Lyapunov functions were also used to establish that the steady states are globally asymptotically stable. The model was further simulated to ascertain the dynamics of CT infection and performed sensitivity analysis on the basic reproduction number from which it was concluded that the parameter $\beta$ is the most sensitive. Due to high rate of sex workers in mining communities, providing adequate healthcare centers for effective treatment of STIs and jobs for the youth will go a long way to minimize the rate of sexual partners and hence help curb the spread of CT infection in the Municipality and Ghana as a whole.

\section{Recommendation}

It is recommended that young women in Ghana especially those in the rural and mining communities should be educated more on genital infections by public health officials and stakeholders.

The paper suggests an establishment of more Healthcare centers and free or highly subsidised treatment for STIs in all the Healthcare centers especially in rural and mining communities in Ghana to curb the spread of CT infections and other STIs.

The paper further recommends that Mine officials should establish water closet toilet facilities with high hygienic observation measures for settlers in their catchment areas instead of pit-latrines. This would help minimize genital infections especially vaginal discharge and other infections associated with pit- latrine.

\section{Conflict of Interest}

The authors declare that they have no competing interests.

\section{Acknowledgements}

The authors express their appreciation to the Municipal Health Directorate and the doctors at Tarkwa Nsuaem Government hospitals for their immense assistance throughout the research.

\section{References}

[1] A. C. Gerbase, J. T. Rowley, D. H. Heymann, S. F. Berkley, and P. Piot (1998), "Global Prevalence and Incidence Estimates of Selected Curable STDs", Sex Transm Infect, vol. 74, no. 1, pp. 12-16.

[2] C. C. Nyarko, C. Unson, P. K. Nyarko and M. Koduah (2014), "Chlamydia trachomatis prevalence in Ghana-A study at a municipal district in Western Ghana", International Journal of Scientific \& Technology Research, vol 3, no. 1, pp. 163-169.

[3] S. Hussen, D. Wachamo, Z. Yohannes and E. Tadesse (2018), "Prevalence of chlamydia trachomatis infection among reproductive age women in sub Saharan Africa: a systematic review and meta-analysis", BMC infectious diseases, vol. 18, no. 1 , pp. $1-8$

[4] W. E. Stamm (2004), "Chlamydia Screening: Expanding the Scope”, Annals of Internal Medicine, vol. 141, pp. 570-572.

[5] K. A. Appea-Kubi, Y. Shinya, B. Sakyi and T. Kisimoto (2004), "Neisseria Gonorrhea, Chlamydial Trachomatis and Alladium Infection in Antenatal and Gynaecological Patients at Korle-Bu Teaching Hospital, Ghana", Jpg J Infect Dis., vol. 57, pp. 253-256.

[6] G. Deceuninck, C. Asamoah-Adu, N. Khonde, J. Pépin, E. H. Frost, S. Deslandes, A. Asamoah-Adu, V. Bekoe and M. Alary (2000), "Improvement of Clinical Algorithms for the Diagnosis of Neisseria Gonorrhea and Chlamydia Trachomatis by use of Gram-stained Smears Among Female Sex Workers in Accra, Ghana", Sex Trans Dis, vol. 27, no. 7, pp. 401-410.

[7] W. Stamm (1999), "Chlamydia Trachomatis Infections: Progress and Problems", J Infectious Disease, vol. 179, pp. 380-383.

[8] Opoku, B. K. and Sarkodie, Y. A. (2010), "Prevalence of Genital Chlamydia and Gonococcal Infections in at Risk Women in the Kumasi Metropolis, Ghana", Ghana Med Journal, 44 (1): 21-24. 
[9] World Health Organization (WHO) (2011). "Prevalence and incidence of selected sexually transmitted infections, Chlamydia trachomatis, Neisseria gonorrhoeae, Syphilis and trichomonas vaginalis" methods and results used by WHO to generate 2005 estimates", Geneva.

[10] Adachi, K., Nielsen-Saines, K. and Klausner J. D. (2016), "Chlamydia trachomatis infection in pregnancy: the global challenge of preventing adverse pregnancy and infant outcomes in sub-Saharan Africa and Asia", 1-21

[11] Molano, M., Meijer, C. J. L. M., Weiderpass, E., Arslan A., Posso, H. and Franceschi, S. (2005), "The natural course of Chlamydia Trachomatis Infection in Asymptomatic Colombian Women: a 5-year Follow-up Study", J Infect Dis., Vol. 191, pp. 907-916.

[12] Smith, J. S., Franceschi, S. and Van den Brule, A. (2007),
"Cervical Infection with Chlamydia Trachomatis and Neisseria Gonorrhoeae in Women from Ten Areas in Four continents". ISSN: 0148-5717, 2007 Aug; Vol. 34, No. 8, pp. 563-569.

[13] R. Ullah, G. Zaman and S. Islam (2013), "Stability analysis of a general SIR epidemic model", VFAST Transactions on Mathematics, vol. 1, no. 1, pp. 57-61.

[14] Ghana Statistical Services (2013), Population and Housing Census, https://statsghana.gov.gh/gssmain/fileUpload/pressrelease/201 0_PHC_National_Analytical_Report.pdf. Accessed: February 2, 2019.

[15] Sharma S. and Samanta G. P (2014). "Analysis of a Chlamydia Epidemic Model" Journal of Biological System, vol. 22 , no. 4 pp. $713-744$. 